\title{
STABILITY: INDEX AND ORDER IN THE BRAUER GROUP
}

\author{
LAWRENCE J. RISMAN
}

ABSTRACT. A field is stable if for every division algebra $A$ in its Brauer group order of $A=$ index of $A$. Index and order in the Bracer group of a field $F$ with discrete valuation and perfect residue class field $K$ are calculated. Division algebras with specified order and index are constructed.

For $F$ complete, necessary and sufficient conditions for the stability of $F$ are given in terms of the Brauer group of $K$. These results follow. A finite extension of a stable field need not be stable. The power series field $K((x))$ is stable for $K$ a local field. $K((x))$ and $K(x)$ are not stable for $K$ a global field.

Introduction. $A$ field $F$ is stable if for every division algebra $A$ in the Brauer group $\mathrm{Br}(F)$, order $e_{F}(A)=$ index $\delta_{F}(A)$. In general order divides index and they have the same prime factors. Any local or global field is stable by class field theory. Many results concerning subfields and subalgebras of division algebras depend on stability. See, for example, [Sc] and [R].

For a profinite group $G$, the character group $\hat{G}=$ continuous $\operatorname{Hom}(G, Q / Z)$. For a field $F, G(F)=\operatorname{Gal}\left(F_{s} / F\right)$ with $F_{s}$ a separable algebraic closure of $F$. If $L$ is a finite Galois extension of $F$ and $f$ is a character on $\mathrm{Gal}(L / F)$, then $f$ inflates to a character on $G(F)$. These topics are exposed in $[\mathrm{S}]$.

Lemma 1. Let $F$ be a field and $G=G(F)$. If $f \in \hat{G}, H=\operatorname{Ker} f$, and $L=$ fixed field of $H$, then $L$ is a finite cyclic extension of $F$ with $\mathrm{Gal}(L / F)=$ $G / H$ and order $f=\operatorname{index}[G: H]=[L: F]$.

If $L$ is a finite Galois extension of $F$, then $L$ is cyclic over $F$ iff there is a character on $\mathrm{Gal}(L / F)$ of order $[L: F]$.

Proof. Immediate from Galois theory and the fact that every finite subgroup of $Q / Z$ is cyclic.

For $M$ a field extension of $F$ and $f \in \hat{G}(F), f_{M}=$ the restriction of $f$ to $G(M)$. This definition assumes $F_{s} \subset M_{s}$. If $f_{M}=0, M$ is said to split $f$. For

Received by the editors March 26, 1974 and, in revised form, May 3, 1974.

AMS (MOS) subject classifications (1970). Primary 16A40, 12B20, 12G05, 12 J10; Secondary 13A20, 10M10. 
$D$ in $\operatorname{Br}(F), D_{M}=D \otimes_{F} M=$ the image of $D$ in $\operatorname{Br}(M)$.

Lemma 2. Let $f \in \hat{G}(F)$ and $L=$ fixed field of $\operatorname{Ker} f$. Let $M$ be a field extension of $F$ and $L M$ a compositum over $F$. Then order $f_{M}=[L M: M], M$ splits $f$ iff $L \subset M$, and order $f_{M}=\operatorname{order} f$ iff $M$ and $L$ are linearly disjoint over $F$.

Proof. This result follows from Galois theory.

Complete fields. Let $K$ be a field complete in a discrete valuation with perfect residue field $\bar{K}$ and $G=G(\bar{K})$. By local field theory [S, Theorem 12.2 , p. 194] we have the following split exact sequence: $0 \rightarrow \mathrm{Br}(\bar{K}) \rightarrow$ $\mathrm{Br}(K) \rightarrow \hat{G} \rightarrow 0$. Hence $\mathrm{Br}(K) \cong \mathrm{Br}(\bar{K}) \oplus \hat{G}$.

Any element of $\operatorname{Br}(K)$ corresponds to a unique $D+f$ with $D \in \operatorname{Br}(\bar{K})$ and $f \in \hat{G}$. By abuse of language we write $D+f \in \operatorname{Br}(K)$.

The embedding of $\hat{G}$ in $\operatorname{Br}(K)$ depends on a choice of a uniformizing parameter $t \in K$. (It does not depend on the completeness of $K_{\text {. }}$ ) Let $f \in \hat{G}$ with $\bar{L}=$ fixed field of $\operatorname{Ker} f$. Let $L$ be the corresponding unramified extension of $K$ with $g$ a generator of $\operatorname{Gal}(L / K)=\operatorname{Gal}(\bar{L} / \bar{K}) . f(g)=m / s \in Q / Z$ with $s=[L: K],(m, s)=1$, and $0<m \leq s$.

The division algebra $A \in \mathrm{Br}(K)$ is defined by the crossed product

$$
Y^{-1} l Y=l^{g}, \quad Y^{s}=t^{m} .
$$

The corresponding factor set in $H^{2}\left(G, K^{*}\right)$ is defined by

$$
c\left(g^{i}, g^{j}\right)= \begin{cases}1 & \text { if } i+j<s \\ t^{m} & \text { if } i+j \geq s .\end{cases}
$$

Proposition 1. Let $D+f$ be in $\operatorname{Br}(K)$ with $e=e-\bar{K}^{(D)}$ and $s=$ order of $f$ in $\hat{G}$. Let $\bar{L}$ be the fixed field of $\operatorname{Ker} f$. Then

1. $e_{k}(D+f)=1$.c.m. $[e, s]$;

2. an unramified extension $M$ of $K$ splits $D+f$ iff $\bar{M}$ splits $D$ and $\bar{L} \subset \bar{M}$;

3. $\delta_{K}(D+f)=$ minimum $[M: K]$ for unramified splitting field $M$ of $D+f$;

4. $\delta_{K}(D+f)=s \cdot \delta_{\bar{L}}\left(D_{\bar{L}}\right)$.

Proof. Assertion 1 is immediate considering $D+f$ in $\operatorname{Br}(\bar{K}) \oplus G$. For $L$ an unramified extension of $K$, the image $(D+f)_{L}$ of $D+f$ in $\operatorname{Br}(L)$ is $D_{\bar{L}}+f_{\bar{L}}$. Assertion 2 then follows by Lemma 2. Assertion 3 follows from the fact that every element of $\mathrm{Br}(K)$ contains an unramified maximal subfield 
[S, Proposition 12.2, p. 191]. Assertion 4 follows from Assertions 2 and 3. Q.E.D.

Corollary 1. If $\bar{K}$ is not stable, then $K$ is not stable.

Proof. By Proposition 1 with $f=0, s=1$.

Corollary 2. If $\operatorname{Br}(\vec{K})=0$, then $K$ is stable, and every element of $\operatorname{Br}(K)$ contains a cyclic unramified maximal subfield which is contained in every unramified splitting field.

Proof. By Proposition 1 with $e=1$.

Proposition 2. $K$ is stable iff $\bar{K}$ is stable and for every cyclic extension $\bar{L}$ of $\bar{K}$ and every division algebra $D$ in $\mathrm{Br}(\bar{K})$, the index reduction factor of $\bar{L}$ and $D=(e, s)$ where $e=\delta_{\bar{K}}(D)$ and $s=[\bar{L}: \bar{K}]$.

Proof. If $K$ is stable, then $\bar{K}$ is stable. Suppose $\bar{K}$ is stable. We show that $K$ is stable iff the above condition holds. Let $D+f$ be in $\operatorname{Br}(K)$. Let $\bar{L}, s$, and $e$ be as in Proposition 1 . Then $\delta \bar{K}(D)=e$, by stability of $\bar{K}$. By Proposition 1,

$$
e_{K}(D+f)=\text { I.c.m. }[e, s]=e s /(e, s) \text {. }
$$

Let $r$ be the index reduction factor of $\bar{L}$ and $D$, so that $\delta_{\bar{L}}\left(D \otimes_{\bar{K}} \bar{L}\right)=$ $e / r$. By Proposition $1, \delta_{K}(D+f)=e s / r$. Hence $e_{K}(D+f)=\delta_{K}(D+f)$ iff $r=(e, s)$. The result follows by Lemma 1. Q.E.D.

Corollary 1. If $\bar{K}$ is a local field of characteristic zero, then $K$ is stable.

Corollary 2. If $\bar{K}$ is a global field of characteristic zero, then $K$ is not stable.

We give an example of a finite extension of a stable field that is not stable. We seek a field $F$ with the following properties.

(1) $F$ is perfect and $\operatorname{Br}(F)=0$.

(2) $K$ is a finite extension of $F$.

(3) $D$ is a division algebra in $\operatorname{Br}(K), d=\delta_{K}(D)$.

(4) $L$ is a cyclic extension of $K, s=[L: K]$.

(5) $D_{L}$ is a division algebra.

(6) $(d, s) \neq 1$.

Given such a field $F$ we consider $F((t))$, the field of formal power series in a variable $t . K((t))$ is a finite extension of $F((t))$. By Proposition 2, $F((t))$ is stable and $K((t))$ is not stable. 
The following example, due to Brumer and Auslander, satisfies the above conditions. Let $\bar{F}$ be the maximum solvable extension of the rationals. Let $\bar{K}$ be a finite extension of $\bar{F}$, obtained by solving an unsolvable equation, with $\bar{L}$ and $\bar{M}$ linearly disjoint cyclic extensions of $\bar{K}, s=[\bar{L}: \bar{K}]$, $d=[\bar{M}: \bar{K}]$, and $(d, s) \neq 1$.

Let $F=\bar{F}((x))$ and $K=\bar{K}((x))$, fields of formal power series in a variable $x$. Let division algebra $D$ in $\mathrm{Br}(K)$ be defined by a character of order $d$ on $\operatorname{Gal}(\bar{M} / \bar{K})$. Let $L=\bar{L}((x))$. By Proposition $1, F$ has the desired properties.

Rational function fields. Let $K$ be a field and $K(x)$ the field of rational functions in a variable $x$.

Lemma 3. The restriction map $\mathrm{Br}(K) \rightarrow \mathrm{Br}(K(x)), D \mapsto D_{K(x)}=D \otimes_{K} K(x)$, is an injective homomorphism of groups. If $D$ is a division algebra in $\mathrm{Br}(K)$, then $D_{K(x)}$ is a division algebra whose order and index in $\mathrm{Br}(K(x))$ are, respectively, equal to those of $D$ in $\mathrm{Br}(K)$.

Proof. The map $D \rightarrow D_{K(x)}$ is clearly a homomorphism. It is injective and preserves index since zero divisors in $D_{K(x)}$ yield by specialization zero divisors in $D$. An injective homomorphism of groups preserves order. Q.E.D.

Corollary. If $K$ is not stable, then $K(x)$ is not stable.

By abuse of language we write $D$ for $D_{K(x)}$.

Let $K$ be a perfect field and $G=G(K)$. Let $t$ be a monic linear polynomial in $K[x]$. For $f$ in $\hat{G}$ with $\bar{L}=$ the fixed field of $\operatorname{Ker} f$, let $L=\bar{L}(x)$. With $L$ so chosen, $A_{f}$ is defined as above in the complete case.

Lemma 4. The map $\hat{G} \rightarrow \operatorname{Br}(K(x))$ given by $f \mapsto A_{f}$ is an injective homomorphism of groups. If $f$ is in $\hat{G}$ then $A_{f}$ is a division algebra in $\operatorname{Br}(K(x))$ and $e_{K(x)}\left(A_{f}\right)=\delta_{K(x)}\left(A_{f}\right)=$ order of $f$ in $\hat{G}$.

Proof. The result follows from the following commutative diagram.

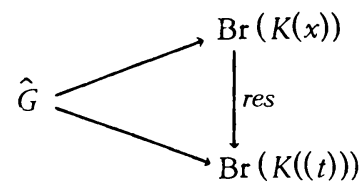

Q.E.D.

By abuse of language we write $f \in \operatorname{Br}(K(x))$. 
Proposition 3. Let $K$ be a perfect field and $G=G(K)$. Then $\operatorname{Br}(K) \oplus \hat{G}$ is isomorphic to a direct summand of $\mathrm{Br}(K(x))$, and the calculations of order and index in $\mathrm{Br}(K) \oplus \hat{G}$ contained in Proposition 1 are valid for $\operatorname{Br}(K(x))$.

Proof. Let $t$ be a monic linear polynomial in $K[x]$. The result follows from the following commutative diagram and the above lemmata.

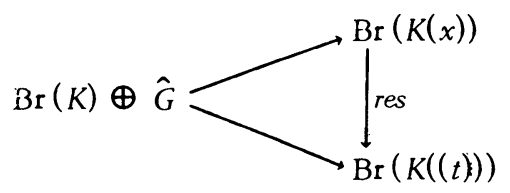

Q.E.D.

Corollary. If $K$ is a global field of characteristic zero, then $K(x)$ is not stable.

Definition. For $K$ a field and $K\left(x_{1}, \cdots, x_{n}\right)$ the field of rational functions in $n$ independent indeterminates, let $G_{1}=G(K)$ and $G_{n}=$ $G\left(K\left(x_{1}, \cdots, x_{n-1}\right)\right)$.

Lemma 5. For all $n, \hat{G}_{n}$ is isomorphic to a subgroup of $\hat{G}_{n+1}$.

Proof. Immediate from Galois theory.

Proposition 4. Let $K$ be a field of characteristic zero.

(1) $\operatorname{Br}(K) \oplus\left(\bigoplus_{i=1}^{n} \hat{G}_{i}\right)$ is a direct summand of $\operatorname{Br}\left(K\left(x_{1}, \cdots, x_{n}\right)\right)$.

(2) $\operatorname{Br}(K) \oplus\left(\bigoplus_{i=1}^{n} \hat{G}(K)\right)$ is isomorphic to a subgroup of $\operatorname{Br}\left(K\left(x_{1}, \cdots, x_{n}\right)\right)$.

Proof. Assertion 1 follows from Proposition 3 by induction. Assertion 2 follows from Assertion 1 and Lemma 5

Definition. For $K$ a field $K_{n}$ is defined inductively by $K_{0}=K$, $K_{n}=K_{n-1}\left(\left(t_{n}\right)\right)$ the field of formal power series in a variable $t_{n} \cdot H_{1}=G(K)$ and $H_{n}=G\left(K_{n-1}\right)$.

Lemma 6. For all $n, \hat{H}_{n}$ is isomorphic to a subgroup of $\hat{H}_{n+1}$.

Proof. Immediate from Galois theory.

Proposition 5. Let $K$ be a field of characteristic zero.

(1) $\operatorname{Br}(K) \oplus\left(\bigoplus_{i=1}^{n} \hat{H}_{i}\right)$ is isomorphic to $\operatorname{Br}\left(K_{n}\right)$.

(2) $\operatorname{Br}(K) \oplus\left(\bigoplus_{i=1}^{n} \hat{G}(K)\right)$ is isomorphic to a subgroup of $\operatorname{Br}\left(K_{n}\right)$.

Proof. Assertion 1 follows from [S, Theorem 12.4, p. 194] by induction. Assertion 2 follows from Assertion 1 by Lemma 6. Q.E.D. 
Index and order. Let $K$ be a field of characteristic zero. For $D \in \operatorname{Br}(K)$ and $L_{1}, \cdots, L_{n}$ cyclic extensions of $K$, consider $D+f_{1}+\cdots+f_{n}$ in $\operatorname{Br}\left(K\left(x_{1}, \cdots, x_{n}\right)\right)$ or $\operatorname{Br}\left(K_{n}\right)$ by Propositions 4 or 5 , where $f_{i} \in \hat{G}(K)$ with $L_{i}=$ fixed field of Kernel $f_{i}$.

Proposition 6. The order of $D+f_{1}+\cdots+f_{n}=$ l.c.m. $\left[e, s_{1}, \cdots, s_{n}\right]$ where $e=e_{K}(D)$ and $s_{i}=\left[L_{i}: K\right]$.

Proof. By Lemma 1 , order $f_{i}=s_{i}$. The result is immediate considering $D+f_{1}+\cdots+f_{n}$ as an element in the direct sum $\operatorname{Br}(K) \oplus\left(\bigoplus_{i=1}^{n} \hat{G}(K)\right)$. Q. E. D.

Proposition 7. Let $L=$ compositum $L_{1} \cdots L_{n}$ over $K$. The index of $D+f_{1}+\cdots+f_{n}=s \cdot d$ where $s=[L: K]$ and $d=\delta_{L}\left(D_{L}\right)$.

Proof. The calculation proceeds by repeated application of Proposition 1. We simplify notation by writing $L_{i}$ in place of $L_{i}\left(x_{1}, \ldots, x_{j}\right)$ or $L_{i j}$.

$$
\begin{aligned}
\delta\left(D+f_{1}+\cdots+f_{n}\right) & =\left[L_{n}: K\right] \delta_{L_{n}}\left(D+f_{1}+\cdots+f_{n-1}\right)_{L_{n}} \quad \text { (by Proposition 1) } \\
& =\left[L_{n}: K\right] \delta_{L_{n}}\left(\left(D+f_{1}+\cdots+f_{n-2}\right)_{L_{n}}+f_{n-1}\right) \\
& =\left[L_{n}: K\right]\left[L_{n-1} L_{n}: L_{n}\right] \delta_{L_{n-1} L_{n}}\left(D+f_{1}+\cdots+f_{n-2}\right) L_{n-1} L_{n} \\
& \vdots \\
& :[L: K] \delta_{L}\left(D_{L}\right) \\
& =s \cdot d . \quad \text { Q.E.D. Proposition 1) }
\end{aligned}
$$

By the above results, exhibiting a division algebra with specified index and order in the Brauer group of $K\left(x_{1}, \ldots, x_{n}\right)$ or $K_{n}$ is reduced, essentially, to finding certain cyclic extensions of $K$.

Proposition 8. Let $K$ be a global field of characteristic zero. Let a and $b$ be positive integers such that $a \mid b$ and $b \mid a^{n}$. Then there exists an element in $\operatorname{Br}\left(K\left(x_{1}, \cdots, x_{n-1}\right)\right)$ and in $\operatorname{Br}\left(K_{n-1}\right)$ with order $=a$ and index $=b$.

Proof. Let $s_{1}, \cdots, s_{n}$ be $n$ integers whose product is $b$ and whose least common multiple is $a$. Let $D$ be a division algebra in $\operatorname{Br}(K)$ with $\delta_{K}(D)=e_{K}(D)=s_{n}$. Let $L_{1}, \cdots, L_{n-1}$ be linearly disjoint cyclic extensions of $K$ and $L=$ compositum $L_{1} \cdots L_{n-1}$ over $K$ such that degree $\left[L_{i}: K\right]=s_{i}$ and $\delta_{L}\left(D_{L}\right)=s_{n}$. The existence of $D$ and the $L_{i}$ is a consequence of the structure of $\mathrm{Br}(K)$ and the Grunwald-Wang theorem [A, Chapter 9]. Let $f_{i} \in$ $\hat{G}(K)$ with $L_{i}=$ fixed field of Kernel $f_{i}$. 
Then $D+f_{1}+\cdots+f_{n-1}$ has order $a$ by Proposition 6 and index $b$ by Proposition 7. Q.E.D.

If $n$ is the smallest integer such that $b \mid a^{n}$, then $n \leq \log _{2} b$. In Brauer's original paper [B] a division algebra with specified order $a$ and index $b$ is constructed over a function field of transcendence degree $b$. In the above corollary we extend this result to the field of all rational functions in fewer variables. The division algebra in [B] is cyclic. While the above division algebra is a crossed product I do not know whether it is cyclic.

Acknowledgment. I wish to thank John Tate for his advice in this work.

\section{REFERENCES}

A. A. Adrian Albert, Structure of algebras, Amer. Math. Soc. Colloq. Publ., vol. 24, Amer. Math. Soc., Providence, R.I., 1961. MR 23 \#A912.

B. Richard Brauer, Über den Index und den Exponenten von Divisionsalgebren, Tôhoku Math. J. 37 (1933), 77-87.

R. Lawrence Risman, Subalgebras of division algebras, Ph.D. Dissertation, Harvard University, Cambridge Mass., 1973.

S. Jean-Pierre Serre, Corps locaux, Hermann, Paris, 1968.

Sc. Murray Schacher, Subfields of division rings. I, J. Algebra 9 (1968), 451477. MR 37 \#2809.

DEPARTMENT OF MATHEMATICS, TECHNION, ISRAEL INSTITUTE OF TECHNOLOGY, HAIFA, ISR AEL 